\title{
Subclasses of Harmonic Mappings Defined by Convolution
}

\author{
Santosh B. Joshi ${ }^{1}$ and Girish D. Shelake ${ }^{2}$ \\ ${ }^{1}$ Department of Mathematics, Walchand College, Sangli, Maharashtra 416415, India \\ ${ }^{2}$ Department of Mathematics, Willingdon College, Sangli, Maharashtra 416415, India
}

Correspondence should be addressed to Santosh B. Joshi; joshisb@hotmail.com

Received 29 November 2012; Accepted 27 February 2013

Academic Editor: Vladislav Kravchenko

Copyright (C) 2013 S. B. Joshi and G. D. Shelake. This is an open access article distributed under the Creative Commons Attribution License, which permits unrestricted use, distribution, and reproduction in any medium, provided the original work is properly cited.

Two new subclasses of harmonic univalent functions defined by using convolution and integral convolution are introduced. These subclasses generate several known and new subclasses of harmonic univalent functions as special cases and provide a unified treatment in the study of these classes. Coefficient bounds, extreme points, distortion bounds, convolution conditions, and convex combination are also determined.

\section{Introduction}

A continuous function $f$ is said to be a complex-valued harmonic function in a simply connected domain $D$ in complex plane $\mathbb{C}$ if both real part of $f$ and imaginary part of $f$ are real harmonic in $D$. Such functions can be expressed as

$$
f=h+\bar{g}
$$

where $h$ and $g$ are analytic in $D$. We call $h$ the analytic part and $g$ the coanalytic part of $f$. A necessary and sufficient condition for $f$ to be locally univalent and sense-preserving in $D$ is that $\left|h^{\prime}(z)\right|>\left|g^{\prime}(z)\right|$ for all $z$ in $D$, see [1].

Every harmonic function $f=h+\bar{g}$ is uniquely determined by the coefficients of power series expansions in the unit disk $U=\{z:|z|<1\}$ given by

$$
h(z)=z+\sum_{n=2}^{\infty} a_{n} z^{n}, \quad g(z)=\sum_{n=1}^{\infty} b_{n} z^{n}, \quad z \in U,\left|b_{1}\right|<1,
$$

where $a_{n} \in \mathbb{C}$ for $n=2,3,4, \ldots$ and $b_{n} \in \mathbb{C}$ for $n=1,2,3, \ldots$. For further information about these mappings, one may refer to $[1-5]$.

In 1984, Clunie and Sheil-Small [1] studied the family $S_{H}$ of all univalent sense-preserving harmonic functions $f$ of the form (1) in $U$, such that $h$ and $g$ are represented by (2). Note that $S_{H}$ reduces to the well-known family $S$, the class of all normalized analytic univalent functions $h$ given in (2), whenever the coanalytic part $g$ of $f$ is zero. Let $K$ and $K_{H}$ denote the respective subclasses of $S$ and $S_{H}$ where the images of $f(U)$ are convex. Denote by $S_{H}^{0}$ the subclass of $S_{H}$ for which $g^{\prime}(0)=0$.

The convolution of two functions of the form

$$
\Phi(z)=z+\sum_{n=2}^{\infty} \mu_{n} z^{n}, \quad \Psi(z)=z+\sum_{n=2}^{\infty} v_{n} z^{n}
$$

is given by

$$
(\Phi * \Psi)(z)=\Phi(z) * \Psi(z)=z+\sum_{n=2}^{\infty} \mu_{n} \nu_{n} z^{n},
$$

and the integral convolution is defined by

$$
(\Phi \diamond \Psi)(z)=\Phi(z) \diamond \Psi(z)=z+\sum_{n=2}^{\infty} \frac{\mu_{n} \nu_{n}}{n} z^{n} .
$$

Towards the end of the last century, Jahangiri [3], Silverman [4], and Silverman and Silvia [5] were amongst those who focused on the harmonic starlike functions. Later Öztürk et. al. [6] defined the class $S_{H}^{*}(\lambda, \alpha)$ consisting of functions $f=$ $h+\bar{g}$ such that $h$ and $g$ are of the forms

$$
h(z)=z-\sum_{n=2}^{\infty}\left|a_{n}\right| z^{n}, \quad g(z)=\sum_{n=1}^{\infty}\left|b_{n}\right| z^{n},
$$


which satisfy the condition

$$
\operatorname{Re}\left\{\frac{z h^{\prime}(z)-\overline{z g^{\prime}(z)}}{\lambda\left(z h^{\prime}(z)-\overline{z g^{\prime}(z)}\right)+(1-\lambda)(h(z)+\overline{g(z)})}\right\}>\alpha
$$

for some $0 \leq \alpha<1,0 \leq \lambda \leq 1$ and for all $z \in U$.

Several authors [3-16] have investigated various subclasses of harmonic functions. In this work, we introduce a new subclass of harmonic functions defined by convolution.

Let $\sigma$ be a real constant with $|\sigma|=1$, then we denote $S_{H}^{0}(\Phi, \Psi, \sigma, \lambda, \alpha)$, the subclass of $S_{H}^{0}$ of functions of the form $f=h+\bar{g} \in S_{H}^{0}$ that satisfy the condition

$$
\operatorname{Re}\left\{\frac{H(f, \Phi, \Psi)}{\lambda H(f, \Phi, \Psi)+(1-\lambda) G(f, \Phi, \Psi)}\right\}>\alpha,
$$

where $H(f, \Phi, \Psi)=h(z) * \Phi(z)-\sigma \overline{g(z) * \Psi(z)}, G(f, \Phi, \Psi)=$ $h(z) \diamond \Phi(z)+\sigma \overline{g(z) \diamond \Psi(z)}, 0 \leq \alpha<1,0 \leq \lambda \leq 1$, and $\Phi, \Psi$ are as given in (3).

We also denote $S P_{H}^{0}(\Phi, \Psi, \sigma, \lambda, \alpha)$, the subclass of $S_{H}^{0}$ of functions of the form $f=h+\bar{g} \in S_{H}^{0}$ that satisfy the condition

$$
\begin{aligned}
\operatorname{Re}\left\{\left(1+e^{i \gamma}\right) \frac{H(f, \Phi, \Psi)}{\lambda H(f, \Phi, \Psi)+(1-\lambda) G(f, \Phi, \Psi)}\right. \\
\left.-e^{i \gamma}\right\}>\alpha,
\end{aligned}
$$

where $\gamma$ is real.

We note that the families $S_{H}^{0}(\Phi, \Psi, \sigma, \lambda, \alpha)$ and $S P_{H}^{0}(\Phi, \Psi$, $\sigma, \lambda, \alpha)$ are of special interest, because they contain various classes of well-known harmonic univalent functions as well as many new ones. For different choice of $\Phi, \Psi, \lambda$, and $\alpha$ we obtain the following various classes introduced by other authors:

(1) $S_{H}^{0}\left(\left(z /(1-z)^{2}\right),\left(z /(1-z)^{2}\right), 1, \lambda, \alpha\right)=S_{H}^{0}(\lambda, \alpha)$ (see Öztürk et. al [6]).

(2) $S_{H}^{0}\left(\left(z /(1-z)^{2}\right),\left(z /(1-z)^{2}\right), 1,0, \alpha\right)=S_{H}^{0}(\alpha)$ (see Jahangiri [3]).

(3) $S_{H}^{0}\left(\left(z /(1-z)^{2}\right),\left(z /(1-z)^{2}\right), 1,0,0\right)=S_{H}^{0}$ (see Silverman and Silvia [5]).

(4) $S_{H}^{0}\left(\left(z /(1-z)^{2}\right),\left(z /(1-z)^{2}\right), 1,0,0\right)=S_{H}^{* 0}$, with $b_{1}=0$ (see Avc1 and Złotkiewicz [17] and Silverman [4]).

(5) $S_{H}^{0}\left(\left(\left(z+z^{2}\right) /(1-z)^{3}\right),\left(\left(z+z^{2}\right) /(1-z)^{3}\right), 1,0, \alpha\right)=$ $K_{H}^{0}(\alpha)$ (see Jahangiri [3]).

(6) $S_{H}^{0}\left(\left(\left(z+z^{2}\right) /(1-z)^{3}\right),\left(\left(z+z^{2}\right) /(1-z)^{3}\right), 1,0,0\right)=K_{H}^{* 0}$ (see Silverman [4]).

(7) $S_{H}^{0}(\Phi, \Psi, 1,0, \alpha)=S_{H}^{0}(\Phi, \Psi, \alpha)$ (see Dixit et al. [11]).

(8) $S_{H}^{0}(\Phi, \Phi, \sigma, 0, \alpha)=S_{H}^{0}(\Phi, \Phi, \alpha)$ (see Ali et al. [7]).
(9) $S_{H}^{0}(\Phi, \Psi, 1, \lambda, \alpha)=S_{H}^{0}(\Phi, \Psi, \lambda, \alpha)$ (see Joshi et al. [14]).

(10) $S_{H}^{0}\left(\Phi, \Psi,(1)^{l}, 0, \alpha\right)=H(l, \alpha)$, where $\mu_{n}=\nu_{n}=n^{l}$ (see Jahangiri et al. [13]).

(11) $S_{H}^{0}\left(\left(z /(1-z)^{\gamma+1}\right),\left(z /(1-z)^{\gamma+1}\right), 1,0, \alpha\right)=R_{H}(\gamma, \alpha)$, where $\gamma>-1$ (see Murugusundaramoorthy [15]).

(12) $S_{H}^{0}\left(\Phi, \Psi,(-1)^{l}, 0, \alpha\right)=M_{H}(l, \gamma, \alpha)$, where $\mu_{n}=v_{n}=$ $n^{l}\left(\left((\gamma+1)_{n-1}\right) /(n-1) !\right)$ and $(\gamma+1)_{n-1}=(\gamma+1)(\gamma+$ 2) $\cdots(\gamma+n-1)$ (see Al-Shaqsi and Darus [10]).

(13) $S_{H}^{0}(\Phi, \Psi, 1, \lambda, \alpha)=R_{H}^{p, q}(\Phi, \Psi, \lambda, \alpha)$, where $\mu_{n}=v_{n}=$ $\left(\left(\left(\gamma_{1}\right)_{n-1} \ldots\left(\gamma_{p}\right)_{n-1}\right) /\left(\left(\beta_{1}\right)_{n-1} \ldots\left(\beta_{p}\right)_{n-1}\right)\right)(1 /(n-1) !)$ (see Murugusundaramoorthy et al. [16]).

It is clear that the class $S_{H}^{0}(\Phi, \Psi, \sigma, \lambda, \alpha)$ generates a number of known subclasses and provides a unified treatment of these subclasses of harmonic mappings. Motivated by work of Ali et al. [7], we obtain convolution characterization for functions in the class $S_{H}^{0}(\Phi, \Psi, \sigma, \lambda, \alpha)$ and $S P_{H}^{0}(\Phi, \Psi, \sigma, \lambda, \alpha)$. We also obtain sufficient coefficient condition for these two classes, and the last section is devoted to determine growth estimates and extreme points for the class $\operatorname{TS}_{H}^{0}(\Phi, \Psi, \sigma, \lambda, \alpha)$ and $\operatorname{TSP}_{H}^{0}(\Phi, \Psi, \sigma, \lambda, \alpha)$.

\section{Main Results}

We now derive a convolution characterization for functions in the class $S_{H}^{0}(\Phi, \Psi, \sigma, \lambda, \alpha)$.

Theorem 1. Let $f=h+\bar{g} \in S_{H}^{0}$. Then $f \in S_{H}^{0}(\Phi, \Psi, \sigma, \lambda, \alpha)$ if and only if

$$
\begin{aligned}
& (h * \Phi) \diamond\left[\frac{z+(((x+2 \alpha-1)(1-\lambda)) /(2-2 \alpha)) z^{2}}{(1-z)^{2}}\right] \\
& -\sigma \overline{(g * \Psi)} \\
& \diamond\left[\left(\frac{x+\alpha-\lambda(x+2 \alpha-1)}{1-\alpha} \bar{z}\right.\right. \\
& \left.\left.\quad-\frac{(x+2 \alpha-1)(1-\lambda)}{2-2 \alpha} \bar{z}^{2}\right)\left((1-\bar{z})^{2}\right)^{-1}\right] \neq 0, \\
& \quad|x|=1,|z| \neq 0 .
\end{aligned}
$$

Proof. A necessary and sufficient condition for $f=h+\bar{g}$ to be in the class $S_{H}^{0}(\Phi, \Psi, \sigma, \lambda, \alpha)$, with $h$ and $g$ of the form (1) is given by (8). The condition (8) holds if and only if

$$
\begin{aligned}
& \frac{1}{(1-\alpha)}\left\{\frac{H(f, \Phi, \Psi)}{\lambda H(f, \Phi, \Psi)+(1-\lambda) \quad G(f, \Phi, \Psi)}-\alpha\right\} \\
& \quad \neq \frac{x-1}{x+1} ; \quad|x|=1, \quad x \neq-1,0<|z|<1 .
\end{aligned}
$$


By simple algebraic manipulation, (11) gives

$$
\begin{gathered}
0 \neq(x+1)[h(z) * \Phi(z)-\sigma \overline{g(z) * \Psi(z)}] \\
-(x+2 \alpha-1)[\lambda(h(z) * \Phi(z)-\sigma \overline{g(z) * \Psi(z)}) \\
+(1-\lambda)(h(z) \diamond \Phi(z) \\
+\sigma \overline{g(z) \diamond \Psi(z))]} \\
=(h * \Phi) \diamond\left[\frac{2(1-\alpha) z+(x-1+2 \alpha)(1-\lambda) z^{2}}{(1-z)^{2}}\right] \\
\left.-\sigma \overline{(g * \Psi)} \frac{2(\bar{x}+\alpha-\lambda(\bar{x}+2 \alpha-1)) z-(\bar{x}+2 \alpha-1)(1-\lambda) z^{2}}{(1-z)^{2}}\right] .
\end{gathered}
$$

The latter condition, along with (8) for $x=-1$, establishes the result for all $|x|=1$.

An application of the convolution condition in Theorem 1 gives sufficient condition for harmonic functions to belong to the class $S_{H}^{0}(\Phi, \Psi, \sigma, \lambda, \alpha)$.

Theorem 2. Let $f=h+\bar{g} \in S_{H}^{0}$. Then $f \in S_{H}^{0}(\Phi, \Psi, \sigma, \lambda, \alpha)$ if

$$
\begin{aligned}
& \sum_{n=2}^{\infty}\left(\frac{n-\alpha-\lambda \alpha(n-1)}{1-\alpha}\right) \frac{\left|\mu_{n}\right|\left|a_{n}\right|}{n} \\
& \quad+\sum_{n=2}^{\infty}\left(\frac{n+\alpha-\lambda \alpha(n+1)}{1-\alpha}\right) \frac{\left|v_{n}\right|\left|b_{n}\right|}{n} \leq 1 .
\end{aligned}
$$

Proof. For $h$ and $g$ given by (2), Theorem 1 gives

$$
\begin{aligned}
& \mid(h * \Phi) \diamond\left[\left(z+\frac{(x+2 \alpha-1)(1-\lambda)}{2-2 \alpha} z^{2}\right)\right. \\
& \left.\times\left((1-z)^{2}\right)^{-1}\right] \\
& -\sigma \overline{(g * \Psi)} \diamond\left[\frac{x+\alpha-\lambda(x+2 \alpha-1)}{1-\alpha} \bar{z}\right. \\
& -\frac{(x+2 \alpha-1)(1-\lambda)}{2-2 \alpha} \bar{z}^{2} \\
& \left.\times\left((1-\bar{z})^{2}\right)^{-1}\right] \mid \\
& =\mid z+\sum_{n=2}^{\infty} \frac{\mu_{n}}{n}\left[n+(n-1) \frac{(x+2 \alpha-1)(1-\lambda)}{2-2 \alpha}\right] a_{n} z^{n} \\
& -\sigma \sum_{n=2}^{\infty} \frac{\nu_{n}}{n}\left[n \frac{x+\alpha-\lambda(x+2 \alpha-1)}{1-\alpha}\right.
\end{aligned}
$$

$$
\begin{array}{r}
\left.-(n-1) \frac{(x+2 \alpha-1)(1-\lambda)}{2-2 \alpha}\right] \overline{b_{n} z^{n} \mid} \\
>|z|\left[1-\sum_{n=2}^{\infty}\left(\frac{n-\alpha-\lambda \alpha(n-1)}{1-\alpha}\right) \frac{\left|\mu_{n}\right|\left|a_{n}\right|}{n}\right. \\
\left.-\sum_{n=2}^{\infty}\left(\frac{n+\alpha-\lambda \alpha(n+1)}{1-\alpha}\right) \frac{\left|v_{n}\right|\left|b_{n}\right|}{n}\right] .
\end{array}
$$

The last expression is nonnegative by hypothesis, and hence by Theorem 1 , it follows that $f \in S_{H}^{0}(\Phi, \Psi, \sigma, \lambda, \alpha)$.

Theorem 3. Let $f=h+\bar{g} \in S_{H}^{0}$. Then $f \in S P_{H}^{0}(\Phi, \Psi, \sigma, \lambda, \alpha)$ if and only if

$$
\begin{aligned}
& (h * \Phi) \diamond\left[z+\frac{\left[(x+1) e^{i \gamma}+x+2 \alpha-1\right](1-\lambda)}{2-2 \alpha} z^{2}\right. \\
& \left.\quad \times\left((1-z)^{2}\right)^{-1}\right] \\
& -\sigma \overline{(g * \Psi)} \\
& \diamond\left[\left(\frac{(x+1) e^{i \gamma}+x+\alpha-\lambda\left((x+1) e^{i \gamma}+x+2 \alpha-1\right)}{1-\alpha} \bar{z}\right.\right. \\
& \left.\left.\quad-\frac{\left[(x+1) e^{i \gamma}+x+2 \alpha-1\right](1-\lambda)}{2-2 \alpha} \bar{z}^{2}\right)\left((1-\bar{z})^{2}\right)^{-1}\right] \neq 0, \\
& \quad|x|=1, \quad|z| \neq 0 .
\end{aligned}
$$

Proof. A necessary and sufficient condition for $f=h+\bar{g}$ to be in the class $S_{H}^{0}(\Phi, \Psi, \sigma, \lambda, \alpha)$, with $h$ and $g$ of the form (1) is given by (9). The condition (9) holds if and only if

$$
\begin{aligned}
\frac{1}{(1-\alpha)}\left\{\left(1+e^{i \gamma}\right) \frac{H(f, \Phi, \Psi)}{\lambda H(f, \Phi, \Psi)+(1-\lambda) G(f, \Phi, \Psi)}\right. \\
\left.-e^{i \gamma}-\alpha\right\} \neq \frac{x-1}{x+1} ; \\
|x|=1, \quad x \neq-1,0<|z|<1 .
\end{aligned}
$$

By simple algebraic manipulation, we get the desired result.

Now sufficient coefficient condition for the class $S P_{H}^{0}(\Phi$, $\Psi, \sigma, \lambda, \alpha)$ is easily obtained.

Theorem 4. Let $f=h+\bar{g} \in S_{H}^{0}$. Then $f \in S_{H}^{0}(\Phi, \Psi, \sigma, \lambda, \alpha)$ if

$$
\begin{aligned}
& \sum_{n=2}^{\infty}\left(\frac{2 n-1-\alpha-\lambda(1+\alpha)(n-1)}{1-\alpha}\right) \frac{\left|\mu_{n}\right|\left|a_{n}\right|}{n} \\
& +\sum_{n=2}^{\infty}\left(\frac{2 n+1+\alpha-\lambda(1+\alpha)(n+1)}{1-\alpha}\right) \frac{\left|v_{n}\right|\left|b_{n}\right|}{n} \leq 1 .
\end{aligned}
$$


We further let $T S_{H}^{0}(\Phi, \Psi, \sigma, \lambda, \alpha)$ and $T S P_{H}^{0}(\Phi, \Psi, \sigma, \lambda, \alpha)$ denote the subclasses of $S_{H}^{0}(\Phi, \Psi, \sigma, \lambda, \alpha)$ and $S P_{H}^{0}(\Phi, \Psi$, $\sigma, \lambda, \alpha)$, respectively, consisting of functions $f=h+\bar{g} \in S_{H}^{0}$ such that $h$ and $g$ are of the form

$$
\begin{aligned}
& h(z)=z-\sum_{n=2}^{\infty} a_{n} z^{n}, \\
& g(z)=\sigma \sum_{n=2}^{\infty} b_{n} z^{n}, \quad a_{n} \geq 0, \quad b_{n} \geq 0 .
\end{aligned}
$$

Let

$$
\Phi(z)=z+\sum_{n=2}^{\infty} \mu_{n} z^{n}, \quad \Psi(z)=z+\sum_{n=2}^{\infty} v_{n} z^{n},
$$

with $\mu_{n} \geq 0, v_{n} \geq 0$.

Theorem 5. Let $f=h+\bar{g}$ of the form (18). Then $f \in T S_{H}^{0}(\Phi, \Psi$, $\sigma, \lambda, \alpha)$ if and only if

$$
\begin{aligned}
& \sum_{n=2}^{\infty} \frac{\mu_{n}}{n}\left(\frac{n-\alpha-\lambda \alpha(n-1)}{1-\alpha}\right) a_{n} \\
& +\sum_{n=2}^{\infty} \frac{\nu_{n}}{n}\left(\frac{n+\alpha-\lambda \alpha(n+1)}{1-\alpha}\right) b_{n} \leq 1 .
\end{aligned}
$$

Proof. If $f \in T S_{H}^{0}(\Phi, \Psi, \sigma, \lambda, \alpha)$, then (8) is equivalent to

$$
\begin{gathered}
\operatorname{Re}\left\{\left((1-\alpha) z-\sum_{n=2}^{\infty} \mu_{n} \frac{[n-\alpha-\alpha \lambda(n-1)]}{n} a_{n} z^{n}\right.\right. \\
\left.-\sum_{n=2}^{\infty} v_{n} \frac{[n+\alpha-\alpha \lambda(n+1)]}{n} b_{n} \bar{z}^{n}\right) \\
\quad \times\left(z-\sum_{n=2}^{\infty} \mu_{n}\left[\lambda+\left(\frac{1-\lambda}{n}\right)\right] a_{n} z^{n}\right. \\
\left.\left.\quad+\sum_{n=2}^{\infty} v_{n}\left[\left(\frac{1-\lambda}{n}\right)-\lambda\right] b_{n} \bar{z}^{n}\right)^{-1}\right\}>0,
\end{gathered}
$$

for $z \in U$. Letting $z \rightarrow 1^{-}$through real values, we get

$$
\begin{gathered}
\left((1-\alpha)-\sum_{n=2}^{\infty} \mu_{n}\left(\frac{[n-\alpha-\alpha \lambda(n-1)]}{n}\right) a_{n}\right. \\
\left.-\sum_{n=2}^{\infty} v_{n}\left(\frac{[n+\alpha-\alpha \lambda(n+1)]}{n}\right) b_{n}\right) \\
\times\left(1-\sum_{n=2}^{\infty} \mu_{n}\left[\lambda+\left(\frac{1-\lambda}{n}\right)\right] a_{n}\right. \\
\left.+\sum_{n=2}^{\infty} v_{n}\left[\left(\frac{1-\lambda}{n}\right)-\lambda\right] b_{n}\right)^{-1} \geq 0,
\end{gathered}
$$

which gives required condition (20).
Conversely, for $h$ and $g$ given by (18),

$$
\begin{aligned}
& \mid(h * \Phi) \diamond[\left(z+\frac{(x+2 \alpha-1)(1-\lambda)}{2-2 \alpha} z^{2}\right) \\
&\left.\times\left((1-z)^{2}\right)^{-1}\right] \\
&-\sigma \overline{(g * \Psi)} \diamond {\left[\left(\frac{x+\alpha-\lambda(x+2 \alpha-1)}{1-\alpha} \bar{z}\right.\right.} \\
&\left.-\frac{(x+2 \alpha-1)(1-\lambda)}{2-2 \alpha} \bar{z}^{2}\right) \\
&\left.\times\left((1-\bar{z})^{2}\right)^{-1}\right]
\end{aligned}
$$

$$
\begin{aligned}
>|z|[ & -\sum_{n=2}^{\infty} \frac{\mu_{n}}{n}\left(\frac{n-\alpha-\lambda \alpha(n-1)}{1-\alpha}\right) a_{n} \\
& \left.-\sum_{n=2}^{\infty} \frac{v_{n}}{n}\left(\frac{n+\alpha-\lambda \alpha(n+1)}{1-\alpha}\right) b_{n}\right],
\end{aligned}
$$

which is nonnegative by hypothesis.

Theorem 6. Let $f=h+\bar{g}$ of the form (18). Then $f \in \operatorname{TSP}_{H}^{0}(\Phi$, $\Psi, \sigma, \lambda, \alpha)$ if and only if

$$
\begin{aligned}
\sum_{n=2}^{\infty} & \frac{\mu_{n}}{n}\left(\frac{2 n-1-\alpha-\lambda(1+\alpha)(n-1)}{1-\alpha}\right) a_{n} \\
& +\sum_{n=2}^{\infty} \frac{\nu_{n}}{n}\left(\frac{2 n+1+\alpha-\lambda(1+\alpha)(n+1)}{1-\alpha}\right) b_{n} \leq 1 .
\end{aligned}
$$

The following theorem gives the distortion bounds for functions in $T S_{H}^{0}(\Phi, \Psi, \sigma, \lambda, \alpha)$, and $T S P_{H}^{0}(\Phi, \Psi, \sigma, \lambda, \alpha)$ which gives a covering result for the classes $T S_{H}^{0}(\Phi, \Psi, \sigma, \lambda, \alpha)$ and $\operatorname{TSP}_{H}^{0}(\Phi, \Psi, \sigma, \lambda, \alpha)$, respectively.

Theorem 7. Let $f \in T S_{H}^{0}(\Phi, \Psi, \sigma, \lambda, \alpha)$ and $\mu_{n} \geq v_{2}, v_{n} \geq$ $v_{2},(n \geq 2)$, then for $|z|=r<1$, we have

$$
\begin{aligned}
r-\frac{2(1-\alpha)}{(2-\alpha-\lambda \alpha) \nu_{2}} r^{2} & \leq|f(z)| \\
& \leq r+\frac{2(1-\alpha)}{(2-\alpha-\lambda \alpha) \nu_{2}} r^{2}
\end{aligned}
$$

The result is sharp with equality for $f(z)=z-(2(1-\alpha) /(2-$ $\left.\alpha-\lambda \alpha) v_{2}\right) z^{2}$. 
Proof. We have

$$
\begin{aligned}
(2-\alpha-\alpha \lambda) \frac{\nu_{2}}{2} \sum_{n=2}^{\infty}\left(a_{n}+b_{n}\right) \\
\leq \sum_{n=2}^{\infty}[n-\alpha-\lambda \alpha(n-1)] \frac{\mu_{n} a_{n}}{n} \\
\quad+\sum_{n=2}^{\infty}[n+\alpha-\lambda \alpha(n+1)] \frac{\nu_{n} b_{n}}{n} \\
\leq 1-\alpha .
\end{aligned}
$$

Thus,

$$
\begin{aligned}
|f(z)| & =\left|z-\sum_{n=2}^{\infty} a_{n} z^{n}+\sigma \sum_{n=2}^{\infty} b_{n} \bar{z}^{n}\right| \\
& \leq r+r^{2} \sum_{n=2}^{\infty}\left(a_{n}+b_{n}\right) \\
& \leq r+\frac{2(1-\alpha)}{(2-\alpha-\lambda \alpha) \nu_{2}} r^{2} .
\end{aligned}
$$

The proof of left-hand inequality follows in lines similar to that of right-hand side inequality.

Theorem 8. Let $f \in T S P_{H}^{0}(\Phi, \Psi, \sigma, \lambda, \alpha)$ and $\mu_{n} \geq v_{2}, v_{n} \geq$ $v_{2}, \quad(n \geq 2)$, then for $|z|=r<1$, we have

$$
\begin{aligned}
r-\frac{2(1-\alpha)}{(3-\alpha-\lambda(1+\alpha)) \nu_{2}} r^{2} & \leq|f(z)| \\
& \leq r+\frac{2(1-\alpha)}{(3-\alpha-\lambda(1+\alpha)) \nu_{2}} r^{2}
\end{aligned}
$$

The result is sharp with equality for $f(z)=z-(2(1-\alpha) /(3-$ $\left.\alpha-\lambda(1+\alpha)) v_{2}\right) z^{2}$.

Corollary 9. Let $f \in T S_{H}^{0}(\Phi, \Psi, \sigma, \lambda, \alpha)$, then

$$
\left\{w:|w|<1-\frac{2(1-\alpha)}{(2-\alpha-\lambda \alpha) \nu_{2}}\right\} \subset f(U) .
$$

Corollary 10. Let $f \in T S P_{H}^{0}(\Phi, \Psi, \sigma, \lambda, \alpha)$, then

$$
\left\{w:|w|<1-\frac{2(1-\alpha)}{(3-\alpha-\lambda(1+\alpha)) \nu_{2}}\right\} \subset f(U) .
$$

Finally, we determine the extreme points of the class $T S_{H}^{0}(\Phi, \Psi, \sigma, \lambda, \alpha)$, and $T S P_{H}^{0}(\Phi, \Psi, \sigma, \lambda, \alpha)$.

Theorem 11. Let

$$
\begin{gathered}
h_{1}(z)=z, \quad h_{n}(z)=z-\frac{n(1-\alpha)}{[n-\alpha-\lambda \alpha(n-1)] \mu_{n}} z^{n}, \\
g_{n}(z)=z-\frac{n(1-\alpha)}{\sigma[n+\alpha-\lambda \alpha(n+1)] \nu_{n}} \bar{z}^{n} \quad(n=2,3, \ldots) .
\end{gathered}
$$

A function $f \in \operatorname{TS}_{H}^{0}(\Phi, \Psi, \sigma, \lambda, \alpha)$ if and only if $f$ can be expressed in the form

$$
f(z)=\sum_{n=1}^{\infty}\left(\beta_{n} h_{n}+\gamma_{n} g_{n}\right)
$$

where $\beta_{n} \geq 0, \gamma_{n} \geq 0, \beta_{1}=1-\sum_{n=2}^{\infty}\left(\beta_{n}+\gamma_{n}\right)$ and $\gamma_{1}=0$. In particular, the extreme points of $\operatorname{TS}_{H}^{0}(\Phi, \Psi, \sigma, \lambda, \alpha)$ are $\left\{h_{n}\right\}$ and $\left\{g_{n}\right\}$.

Proof. Let

$$
\begin{aligned}
f(z)= & \sum_{n=2}^{\infty}\left(\beta_{n} h_{n}+\gamma_{n} g_{n}\right) \\
= & z-\sum_{n=2}^{\infty} \beta_{n} \frac{n(1-\alpha)}{[n-\alpha-\lambda \alpha(n-1)] \mu_{n}} z^{n} \\
& +\sigma \sum_{n=2}^{\infty} \gamma_{n} \frac{n(1-\alpha)}{[n+\alpha-\lambda \alpha(n+1)] \nu_{n}} \bar{z}^{n} .
\end{aligned}
$$

Since

$$
\begin{aligned}
\sum_{n=2}^{\infty} & \frac{\mu_{n}}{n}\left(\frac{n-\alpha-\lambda \alpha(n-1)}{1-\alpha}\right) \beta_{n} \frac{n(1-\alpha)}{[n-\alpha-\lambda \alpha(n-1)] \mu_{n}} \\
& +\sum_{n=1}^{\infty} \frac{\nu_{n}}{n}\left(\frac{n+\alpha-\lambda \alpha(n+1)}{1-\alpha}\right) \gamma_{n} \frac{n(1-\alpha)}{[n+\alpha-\lambda \alpha(n+1)] \nu_{n}} \\
= & \sum_{n=2}^{\infty}\left(\beta_{n}+\gamma_{n}\right)=1-\beta_{1} \leq 1,
\end{aligned}
$$

from Theorem 5, $f \in T S_{H}^{0}(\Phi, \Psi, \sigma, \lambda, \alpha)$.

Conversely, if $f \in T S_{H}^{0}(\Phi, \Psi, \sigma, \lambda, \alpha)$, then $a_{n} \leq n(1-$ $\alpha) /(n-\alpha-\lambda \alpha(n-1)) \mu_{n}$ and $b_{n} \leq n(1-\alpha) /\left(n+\alpha-\lambda \alpha(n+1) \nu_{n}\right)$. Set $\beta_{n}=\left(\mu_{n} / n\right)(n-\alpha-\lambda \alpha(n-1) /(1-\alpha)) a_{n}, \gamma_{n}=\left(\nu_{n} / n\right)(n+$ $\alpha-\lambda \alpha(n+1) /(1-\alpha)) b_{n}, \beta_{1}=1-\sum_{n=2}^{\infty}\left(\beta_{n}+\gamma_{n}\right)$, and $\gamma_{1}=0$.

Then,

$$
\sum_{n=2}^{\infty}\left(\beta_{n} h_{n}+\gamma_{n} g_{n}\right)=z-\sum_{n=2}^{\infty} a_{n} z^{n}+\sigma \sum_{n=2}^{\infty} b_{n} \bar{z}^{n}=f(z)
$$

Theorem 12. Let

$$
\begin{aligned}
& p_{1}(z)=z, \\
& p_{n}(z)=z-\frac{n(1-\alpha)}{[2 n-1-\alpha-\lambda(1+\alpha)(n-1)] \mu_{n}} z^{n}, \\
& q_{n}(z)=z-\frac{n(1-\alpha)}{\sigma[2 n+1+\alpha-\lambda(1+\alpha)(n+1)] \nu_{n}} \bar{z}^{n}
\end{aligned}
$$


A function $f \in \operatorname{TSP}_{H}^{0}(\Phi, \Psi, \sigma, \lambda, \alpha)$ if and only if $f$ can be expressed in the form

$$
f(z)=\sum_{n=1}^{\infty}\left(\beta_{n} p_{n}+\gamma_{n} q_{n}\right)
$$

where $\beta_{n} \geq 0, \gamma_{n} \geq 0, \beta_{1}=1-\sum_{n=2}^{\infty}\left(\beta_{n}+\gamma_{n}\right)$, and $\gamma_{1}=0$. In particular, the extreme points of $\operatorname{TSP}_{H}^{0}(\Phi, \Psi, \sigma, \lambda, \alpha)$ are $\left\{p_{n}\right\}$ and $\left\{q_{n}\right\}$.

\section{Acknowledgment}

The authors are thankful to the referees for their valuable suggestions and comments.

\section{References}

[1] J. Clunie and T. Sheil-Small, "Harmonic univalent functions," Annales Academiae Scientiarum Fennicae A, vol. 9, pp. 3-25, 1984.

[2] P. Duren, Harmonic Mappings in the Plane, vol. 156 of Cambridge Tracts in Mathematics, Cambridge University Press, Cambridge, UK, 2004.

[3] J. M. Jahangiri, "Harmonic functions starlike in the unit disk," Journal of Mathematical Analysis and Applications, vol. 235, no. 2, pp. 470-477, 1999.

[4] H. Silverman, "Harmonic univalent functions with negative coefficients," Journal of Mathematical Analysis and Applications, vol. 220, no. 1, pp. 283-289, 1998.

[5] H. Silverman and E. M. Silvia, "Subclasses of harmonic univalent functions," New Zealand Journal of Mathematics, vol. 28, no. 2, pp. 275-284, 1999.

[6] M. Öztürk, S. Yalçin, and M. Yamankaradeniz, "Convex subclass of harmonic starlike functions," Applied Mathematics and Computation, vol. 154, no. 2, pp. 449-459, 2004.

[7] R. M. Ali, B. A. Stephen, and K. G. Subramanian, "Subclasses of harmonic mappings defined by convolution," Applied Mathematics Letters, vol. 23, no. 10, pp. 1243-1247, 2010.

[8] R. M. Ali, M. H. Khan, V. Ravichandran, and K. G. Subramanian, "A class of multivalent functions with negative coefficients defined by convolution," Bulletin of the Korean Mathematical Society, vol. 43, no. 1, pp. 179-188, 2006.

[9] O. P. Ahuja, "Planar harmonic univalent and related mappings," Journal of Inequalities in Pure and Applied Mathematics, vol. 6, no. 4, article 122, 18 pages, 2005.

[10] K. Al-Shaqsi and M. Darus, "On harmonic functions defined by derivative operator," Journal of Inequalities and Applications, vol. 2008, Article ID 263413, 10 pages, 2008.

[11] K. K. Dixit, A. L. Pathak, S. Porwal, and R. Agarwal, "On a subclass of harmonic univalent functions defined by convolution and integral convolution," International Journal of Pure and Applied Mathematics, vol. 69, no. 3, pp. 255-264, 2011.

[12] B. A. Frasin, "Comprehensive family of harmonic univalent functions," SUT Journal of Mathematics, vol. 42, no. 1, pp. 145-155, 2006.

[13] J. M. Jahangiri, G. Murugusundaramoorthy, and K. Vijaya, "Sala-gean-type harmonic univalent functions," Southwest Journal of Pure and Applied Mathematics, vol. 2002, no. 2, pp. 77-82, 2002.
[14] S. B. Joshi, G. D. Shelake, and A. H. Suzeini, "On a subclass of harmonic univalent functions defined by convolution," communicated.

[15] G. Murugusundaramoorthy, "A class of Ruscheweyh-type harmonic univalent functions with varying arguments," Southwest Journal of Pure and Applied Mathematics, no. 2, pp. 90-95, 2003.

[16] G. Murugusundaramoorthy, K. Vijaya, and B. A. Frasin, "A subclass of harmonic functions with negative coefficients defined by Dziok-Srivastava operator," Tamkang Journal of Mathematics, vol. 42, no. 4, pp. 463-473, 2011.

[17] Y. Avc1 and E. Złotkiewicz, "On harmonic univalent mappings," Annales Universitatis Mariae Curie-Skłodowska, vol. 44, pp. 1-7, 1990. 


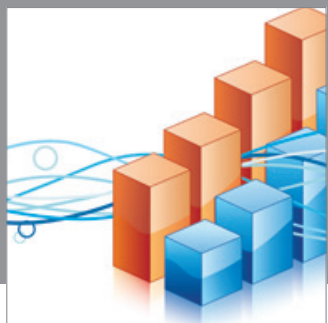

Advances in

Operations Research

mansans

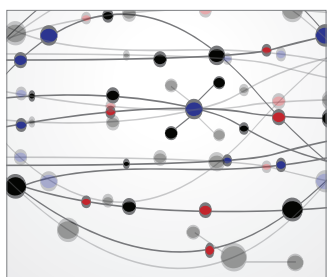

The Scientific World Journal
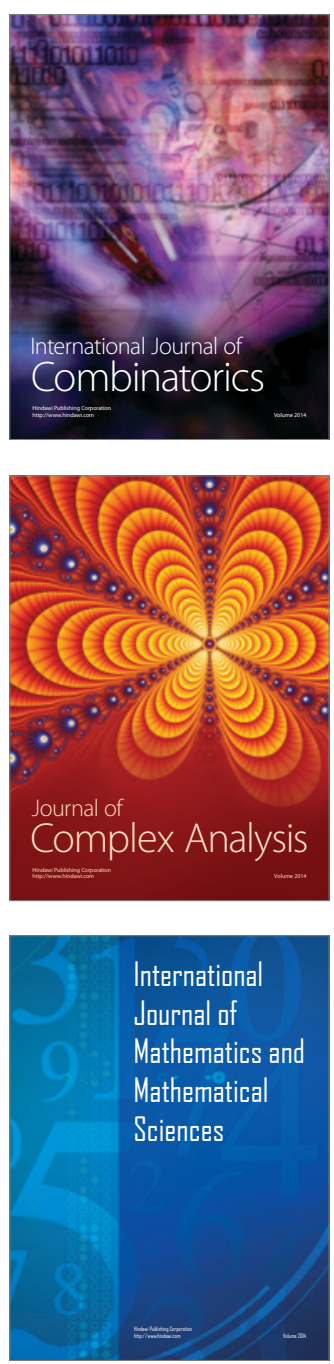
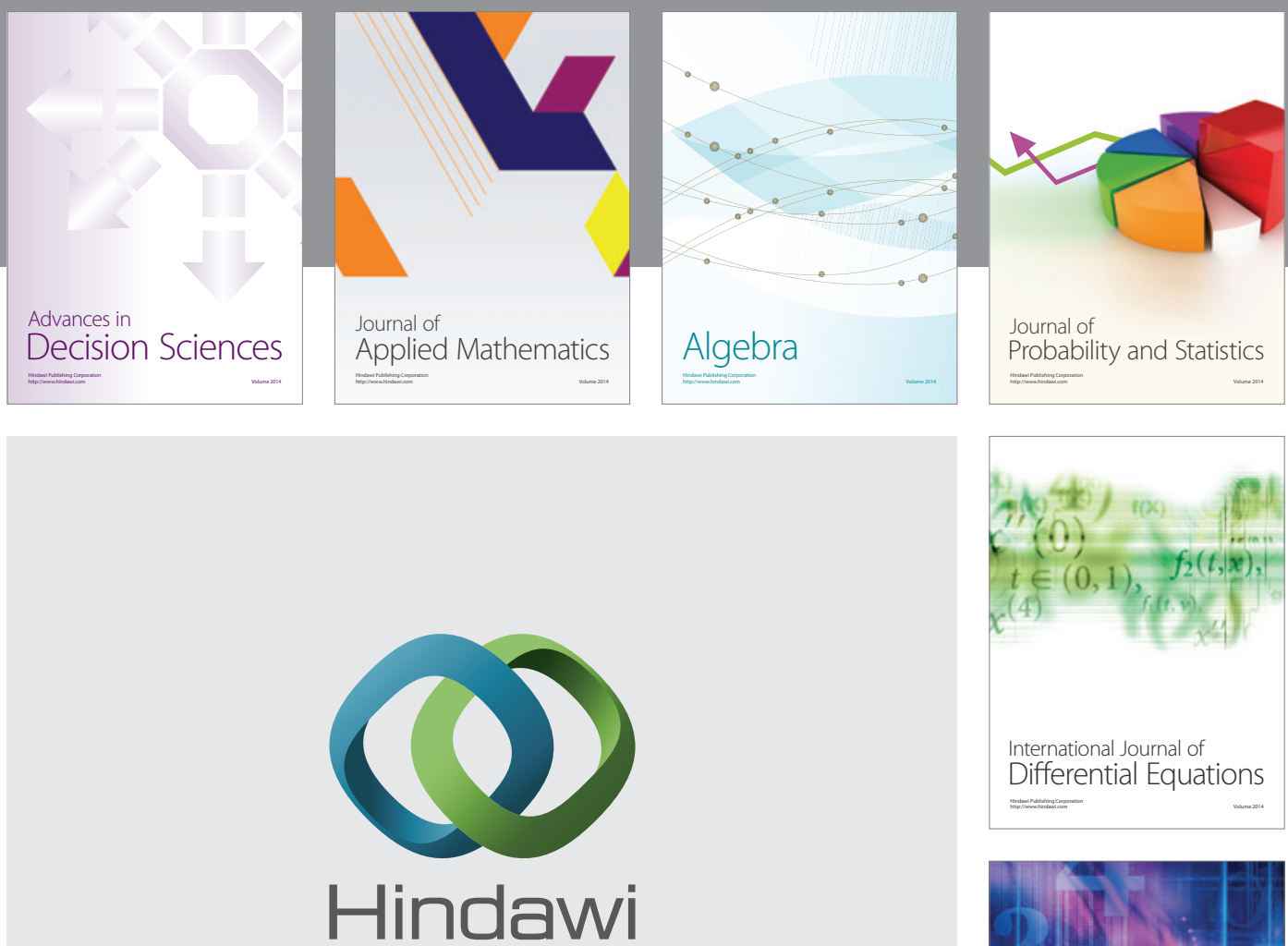

Submit your manuscripts at http://www.hindawi.com
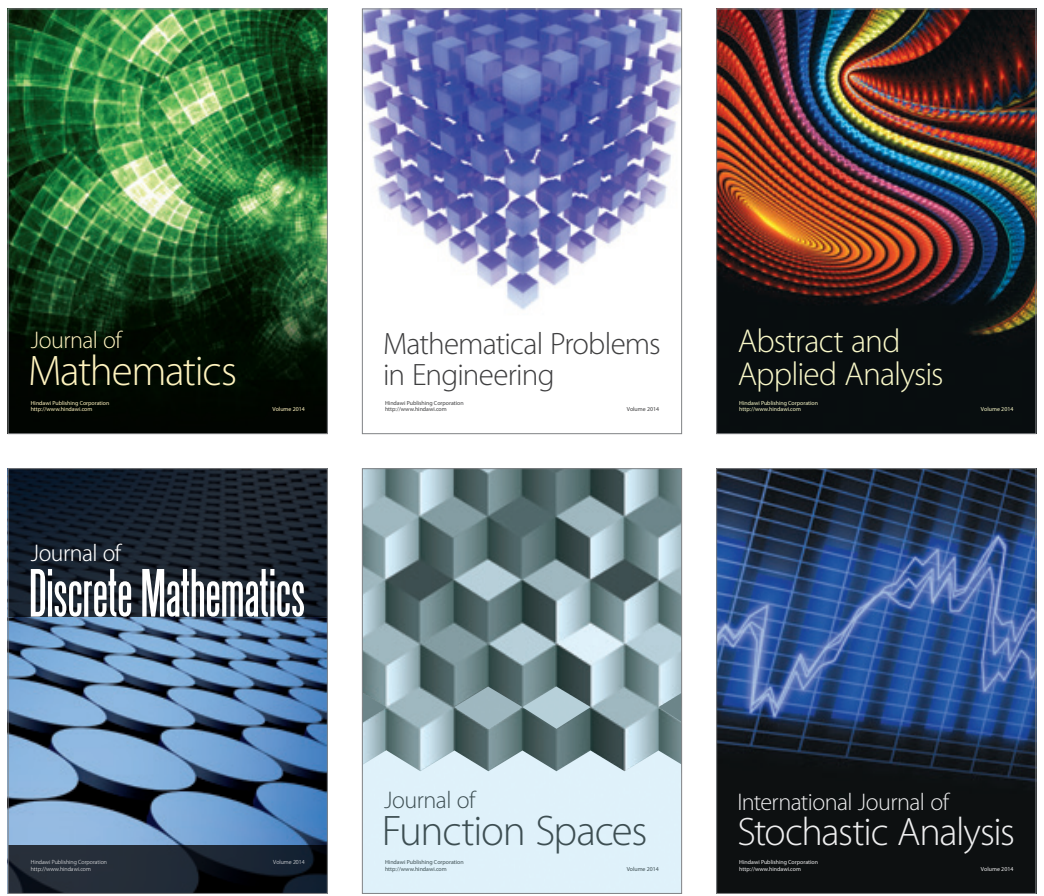

Journal of

Function Spaces

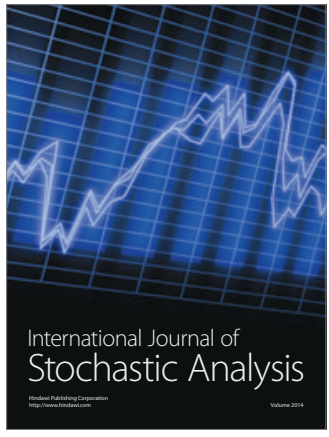

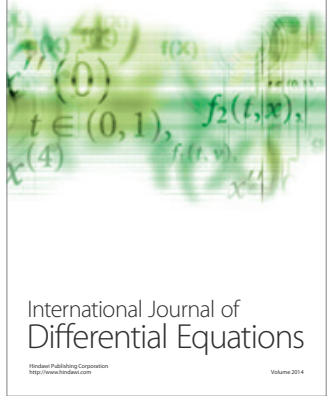
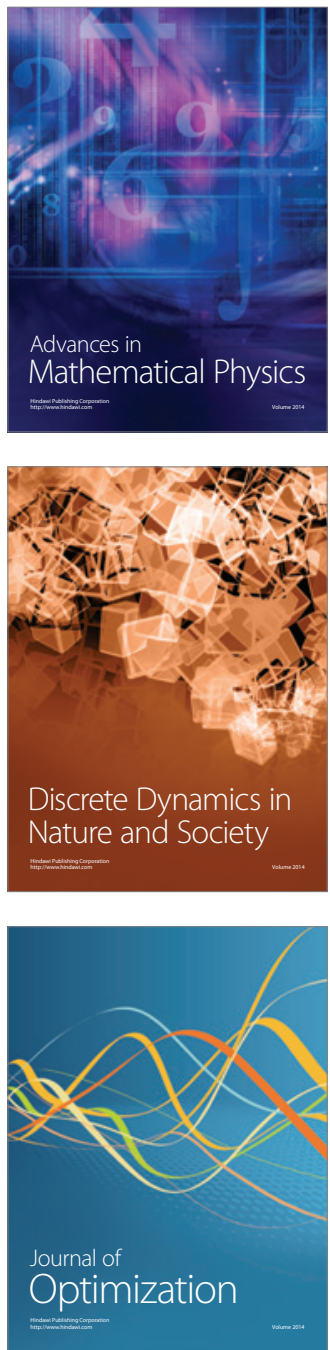